\title{
A new method to estimate point thermal transmittance based on combined two-dimensional heat flow calculation
}

\author{
Jaanus Hallik ${ }^{1, *}$ and Targo Kalamees ${ }^{2,1}$ \\ ${ }^{1}$ nZEB Research Group, Tallinn University of Technology, Estonia \\ ${ }^{2}$ Smart City Center of Excellence (Finest Twins), Tallinn University of Technology, Estonia
}

\begin{abstract}
A well-insulated, airtight and thermal bridge free building envelope is a key factor for nearly zero energy buildings (nZEB). However, increased insulation thickness and minimized air leakages increase the effect of thermal bridges on overall energy efficiency of the nZEBs. Although several more prominent linear thermal bridges are accounted for in the practice the three-dimensional heat flow through vast array of fixation elements, mounting brackets and other point thermal bridges are usually neglected due to time-consuming model preparation routine, lack of input data as well as high number of different thermal bridges that have to be assessed for a single project. In this study a new method was proposed for predicting three-dimensional heat flow and the point thermal transmittance of thermal bridges caused by full or partial penetration of the building envelope with metal elements with uniform geometry in third dimension based on multiple twodimensional numerical heat flow calculations. A new parameter (equivalent length of thermal bridge) was defined which incorporates the effect of additional thermal transmittance in third dimension when multiplied by the difference of two thermal coupling coefficients derived for two-dimensional cross section. Multiple linear regression model was fitted on database with 102 cases and verified with separate case of window to wall connection incorporating metal penetration at fixation points. The proposed methodology can be useful in general practice where the design team lacks the skills or software tools for conducting detailed numerical analysis in three dimensions.
\end{abstract}

\section{Introduction}

A well-insulated, airtight and thermal bridge free building envelope is a key factor for nearly zero energy buildings that becomes mandatory from year 2021. Minimising heat losses and combining a thermally optimised building envelope with the passive use of solar energy allows a significant reduction in the heat load and heating energy demand of residential buildings. However, increased insulation thickness and minimized air leakages increase the effect of thermal bridges on overall heat loss nZEB building envelope. The contribution of thermal bridges to overall heat loss is highly dependent on architectural and structural solution but can easily be as high as $30-40 \%$ of total heat loss of the building envelope [1-5] and influence of moisture safety [6]. Although several more prominent linear thermal bridges are accounted for in the practice the three-dimensional heat flow through vast array of fixation elements and other point thermal bridges are usually neglected. Based on experience with less insulated structures it is usually expected that various point thermal bridges have small contribution to overall heat loss, but several recent studies have shown that within highly insulated building envelope the highly conductive metal fixing elements and cladding systems have considerable effect on effectiveness of thermal insulation layers [7]. Although the detailed numerical calculation methodology for steady state threedimensional heat flow is described in European standard ISO 10211:2017 [8] along with other connected standards it is not implemented in general practice due to timeconsuming model preparation routine, lack of input data as well as high number of different thermal bridges that have to be assessed for a single project. Additionally, the selection of software tools for three-dimensional numerical heat flow modelling is limited due to high price and steep learning curve (ANSYS [9], COMSOL Multiphysics [10], PHYSIBEL Trisco and Solido [11]) compared to numerical heat flow calculation tools in two dimensions where several open source or free to use tools (LBNL Therm [12] etc) are generally available.

Omitting numerical calculation of point thermal transmittances for different point thermal bridges introduces great uncertainty in estimating the overall heat loss of highly insulated building envelope and different parametric or simplified calculation procedures are needed for general practice to describe the overall thermal transfer value of entire building envelope close to reality. There have been several efforts to generalize the thermal bridge effects of different fixation elements inside the insulating layer with sensitivity analysis to show the effect of different technical parameters contributing to heat flow

\footnotetext{
* Corresponding author: jaanus.hallik@taltech.ee
} 
through these thermal bridges such as thermal conductivity of bridging element and surrounding insulation layer, thermal resistances of adjoining layers etc $[7,13,14]$. Additionally several methods have been proposed how to estimate two-dimensional thermal bridge effects from one-dimensional building envelope data, such as thermal transmittances etc $[4,15]$.

A very rough estimation of point thermal transmittance can be made by multiplying the length of thermal bridging element with the heat flow difference per unitary temperature difference derived from two distinct twodimensional calculations. One cross section with thermal bridge element and other without it. This always underestimates the actual three-dimensional heat flow because the heat flow from third dimension to the bridging element is neglected.

Current study proposes a new methodology for estimating the three-dimensional heat flow caused by point thermal bridge based on multiple two-dimensional numerical heat flow calculations by using a multiple regression model to estimate the equivalent length of point thermal bridge based on several parameters related to heat flow through the building envelope.

The hypothesis of the study is that the difference between actual length of thermal bridge and the equivalent length of thermal bridge is strongly correlated to difference in thermal coupling coefficients derived from respective cross sections (i.e. central plane of thermal bridge and reference plane without it) and that difference partially involves the effect of varying geometry and conductivities to the heat flow in the third dimension.

The proposed methodology can be useful in general practice where the design team lacks the skills or software tools for conducting detailed numerical analysis in three dimensions.

\section{Materials and methods}

\subsection{Finite element analysis}

Thermal bridges are defined as part of the building envelope where the otherwise uniform thermal resistance is significantly changed by full or partial penetration of the building envelope by materials with higher thermal conductivity [8]. The point thermal transmittance $(\chi)$, which represents the additional heat flow per $1 \mathrm{~K}$ air temperature difference between the internal and the external environment, of this kind of penetrations is calculated as in equation 1 .

$$
\chi=L_{3 D}-\sum_{i=1}^{N_{i}} U_{i} \cdot A_{i}-\sum_{k=1}^{N_{k}} \psi_{k} \cdot l_{k}
$$

where $L_{3 D}$ is the thermal coupling coefficient obtained from a three-dimensional numerical calculation, $U_{i}$ is the thermal transmittance of the building envelope adjoining the thermal bridge, $A_{i}$ is the respective area of the adjoining part of the building envelope in the calculation model, $\psi_{\mathrm{k}}$ is the linear thermal transmittance of additional thermal bridge if present and $l_{k}$ is the respective length of additional linear thermal bridge.

The linear thermal transmittance of additional thermal bridge is calculated as described in equation 2 .

$$
\psi=L_{2 D}-\sum_{j=1}^{N_{j}} U_{j} \cdot l_{j}
$$

where $L_{2 D}$ is the thermal coupling coefficient obtained from a separate two-dimensional numerical calculation, $U_{j}$ is the thermal transmittance of the building envelope adjoining the thermal bridge and $l_{j}$ is the respective length of adjoining part of the building envelope described in the calculation model.

The point thermal transmittance of assessed thermal bridge can also be derived as in equation 3 when threedimensional reference calculation without assessed penetrating element already contains the effect of additional linear thermal bridge.

$$
\chi=L_{3 D}-L_{3 D r e f}
$$

where $L_{3 D r e f}$ is the thermal coupling coefficient obtained from a separate three-dimensional numerical calculation model with identical boundary conditions, cut-off planes etc, but without considering the presence of assessed point thermal bridge. The thermal coupling coefficients $L_{2 D}$ and $L_{2 D r e f}$ as well as $L_{3 D}$ and $L_{3 D r e f}$ obtained from two- and three-dimensional numerical models are calculated as respective heat flows divided by the temperature difference used in the model.

The heat flow and thermal bridge calculations in this study was carried out according to methodology, boundary conditions and modelling rules described in ISO 10211:2017 using software package Physibel Trisco [11], which utilizes finite difference method to numerically calculate three-dimensional heat flows in complex geometrical models.

For each assessed point thermal bridge case four heat flows were numerically calculated:

- three-dimensional heat flow for actual point thermal bridge;

- three-dimensional reference heat flow for same model omitting the thermal point penetration;

- two-dimensional heat flow for horizontal cross section taken from the central plane of point thermal bridge element and

- two-dimensional reference heat flow for horizontal cross section omitting the thermal point penetration.

To avoid the uncertainty generated by the use of different numerical software tools for two- and threedimensional calculation the two-dimensional heat flow was obtained from three-dimensional calculation model, where third dimension was set to unitary length $(1 \mathrm{~m})$ and modelled with uniform geometry in that dimension. 


\subsection{Description of assessed thermal bridging elements}

In current study an external wall construction with external insulation layer, wind barrier and ventilated cladding with penetrating metal bracket fixed to vertical timber elements was assessed. Schematic horizontal cross sections for thermal bridge cases and reference cases are shown in figure 1.

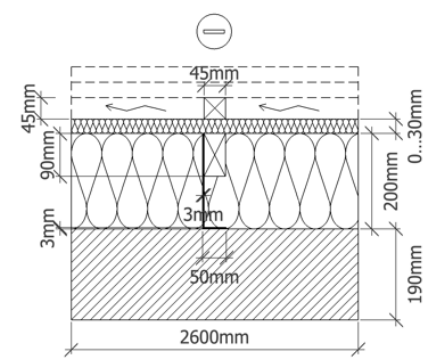

a

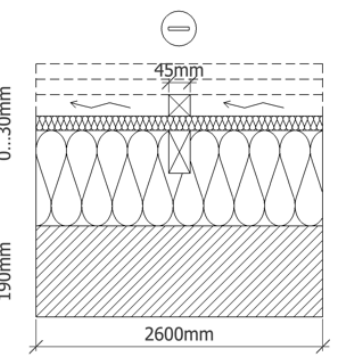

b
Fig. 1. Schematic cross section (horizontal) of assessed external wall for calculating the thermal coupling coefficient $L_{2 D}$ with thermal bridge (a) and thermal coupling coefficient $L_{2 D r e f}$ for reference cases (b) without point thermal bridge.

Previous studies have shown that along with thermal conductivity of penetrating element itself the thermal conductivity of insulation material and its layer thickness used in building envelope as well as the resistances of the layers covering the penetrating element on internal and external sides have significant effect on point thermal transmittance [7,13]. In current study thermal conductivity $(\lambda, \mathrm{W} /(\mathrm{mK}))$ of internal load bearing structure, insulation layer and wind barrier layer along with metal thermal bridging element was varied as described in Table 1. Assessed different insulation materials correspond to two different typical mineral wool types along with polyurethane foam insulation that are used in this kind of construction for nearly zero energy buildings.

Table 1. Variable parameters used in heat flow analysis.

\begin{tabular}{|c|c|c|}
\hline & Description & $\lambda, \mathrm{W} /(\mathrm{mK})$ \\
\hline Load & 0.19 m reinforced concrete & 2.50 \\
bearing & 0.19 m aerated concrete & 0.15 \\
structure & typical mineral wool & 0.040 \\
\hline Insulation & good mineral wool & 0.031 \\
layer & polyurethane foam & 0.023 \\
\hline & 0.009 m gypsum board & 0.250 \\
Wind & 0.030 m gypsum board & 0.250 \\
barrier & 0.030 m mineral wool board & 0.031 \\
& 0.030 m fibreboard & 0.055 \\
& only wind barrier membrane & $(-)$ \\
\hline \multirow{2}{*}{ Metal } & Regular steel & 55.0 \\
bracket & Reinforced steel & 17.0 \\
& Aluminium & 160.0 \\
\hline
\end{tabular}

In addition to variable thermal conductivity and layer thickness the length of metal bracket in third dimension was varied to include brackets with following length: $0.06 \mathrm{~m}, 0.12 \mathrm{~m}$ and $0.18 \mathrm{~m}$. The thickness of metal brackets in all calculated cases was set to $0.003 \mathrm{~m}$. Altogether 102 different combinations were calculated.

The cut-off planes for two- and three-dimensional models were selected according to ISO 20211:2017 to eliminate the effect of thermal bridge on heat flow distribution near the cut-off planes of the calculation model. The total with of two-dimensional calculation model was $2.60 \mathrm{~m}$ while the width and height of the threedimensional calculation model were both $2.60 \mathrm{~m}$ as shown in figure 2 and figure 3.

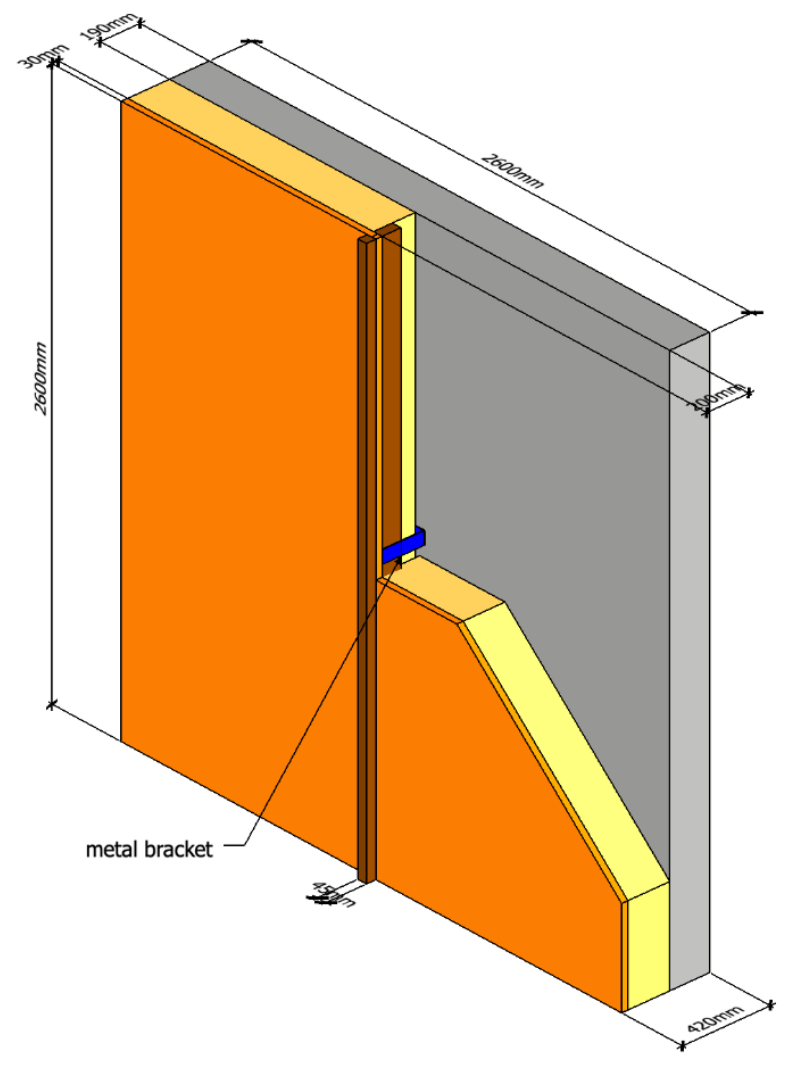

Fig. 2. Geometric model of assessed external wall part containing the thermal bridge (the parts of wind barrier and insulation layers are cut off for visualizing purpose).

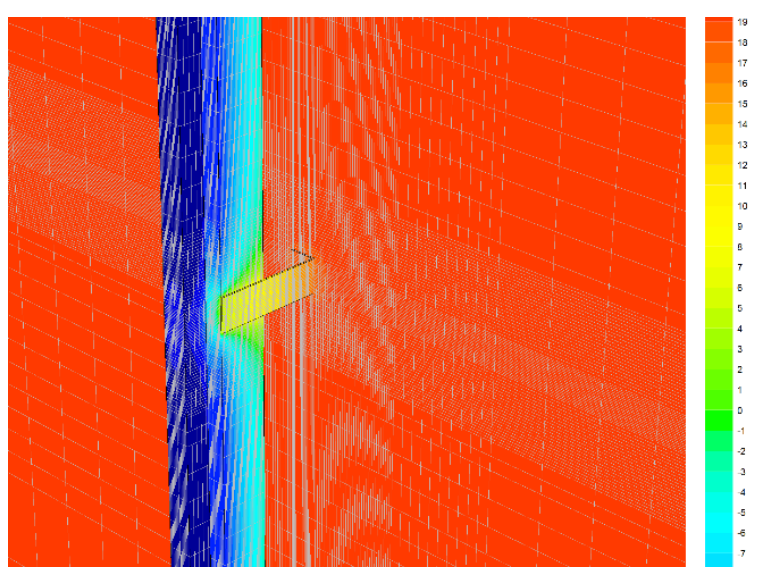

Fig. 3. Three-dimensional FED model of assessed external wall part containing the thermal bridge with indicated mesh density and temperature distribution (the external wind barrier and insulation layers are hidden for visualizing purpose). 
Based on three-dimensional thermal coupling coefficients the point thermal transmittance was calculated as in equation 3 . The acquired point thermal transmittance was divided by the difference of twodimensional thermal coupling coefficients to obtain the equivalent length of the thermal bridge. As the point thermal transmittance of the bridging element is directly related to actual thermal bridge length this effect is omitted from further analysis by subtracting the actual length of thermal bridge $\left(h_{T B}\right)$ from the equivalent length of thermal bridge $\left(h_{e q}\right)$ so the effect of different parameters is tested against the additional length of the thermal bridge $\left(h_{\text {add }}\right)$ as in equation 4.

$$
h_{\text {add }}=h_{e q}-h_{T B}
$$

The half amount of additional length of thermal bridge can be considered as extra length on both side of the bridging element to compensate for the heat flows in third dimension as shown in figure 4.

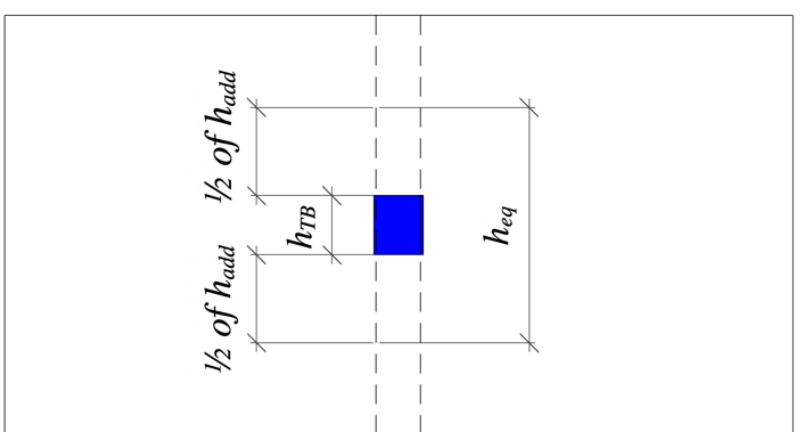

Fig. 4. Schematic cross section (vertical) of assessed external wall with actual length $\left(h_{T B}\right)$, equivalent length $\left(h_{e q}\right)$ and additional length $\left(h_{a d d}\right)$ of thermal bridge in third dimension.

\subsection{Statistical analysis}

The heat flows and thermal coupling coefficients for each combination of input variables were combined into a database. Based on the calculated point thermal transmittances and difference of two-dimensional thermal coupling coefficients several multiple linear regression models were fitted to the numerically calculated data. As first step simple linear regression was fitted as in equation 5 , but additional terms to basic multiple linear regression equation were added to improve the model fit as given in equations 6,7 and 8 based on preliminary numerically calculated data (altogether 12 different equations were fitted, but only 4 most relevant are given here).

$$
\begin{aligned}
& h_{\text {add }}=c_{1} \cdot\left(L_{2 D}-L_{2 D r e f}\right)+c_{n} \\
& h_{\text {add }}=c_{1} \cdot\left(L_{2 D}-L_{2 D r e f}\right)+c_{2} \cdot \lambda_{T B}+c_{3} \cdot R_{i l}+ \\
& +c_{4} \cdot R_{e l}+c_{n} \\
& h_{\text {add }}=c_{1} \cdot\left(L_{2 D}-L_{2 D r e f}\right)+c_{2} \cdot \lambda_{T B}+c_{3} \cdot h_{T B}+ \\
& +c_{4} \cdot R_{e l} \cdot\left(L_{2 D}-L_{2 D r e f}\right)+c_{n} \\
& h_{a d d}=c_{1} \cdot\left(L_{2 D}-L_{2 D r e f}\right)+c_{2} \cdot \lambda_{T B}+c_{3} \cdot h_{T B}+ \\
& +c_{4} \cdot R_{e l} \cdot\left(L_{2 D}-L_{2 D r e f}\right)+c_{5} \cdot R_{i l}+c_{n}
\end{aligned}
$$

where $R_{i l}, R_{e l}$ are the thermal resistances of the layers adjoining the thermal bridging element from internal and external side, $\lambda_{\mathrm{TB}}$ is the thermal conductivity of thermal bridging element, $\mathrm{h}_{\mathrm{tb}}$ is the actual length of thermal bridging element and $\mathrm{c}_{1}, \mathrm{c}_{2}, \mathrm{c}_{3}, \mathrm{c}_{4}, \mathrm{c}_{5}$ and $\mathrm{c}_{\mathrm{n}}$ are the estimated coefficients.

The goodness of fit was assessed by considering the adjusted R-squared for each model and the p-value for each coefficient estimate. For the analysis and model fitting by least squares method the statistical analysis software R (version 3.5.1) [16] was used with several addon packages to allow statistical analysis and data visualisation.

\section{Results}

\subsection{Correlation of main parameters}

Altogether 102 different combination of thermal bridging situations were numerically calculated. For testing the effect of thermal bridge length in third dimension, a concrete wall with regular steel fixation bracket with 60 $\mathrm{mm}$ length combined with three alternative insulation materials and $9 \mathrm{~mm}$ gypsum wind barrier layer was selected as the base case. Other variable parameters were tested in all possible combinations. The correlation between point thermal transmittance and the difference of two-dimensional thermal coupling coefficients can be seen in figure 5 .

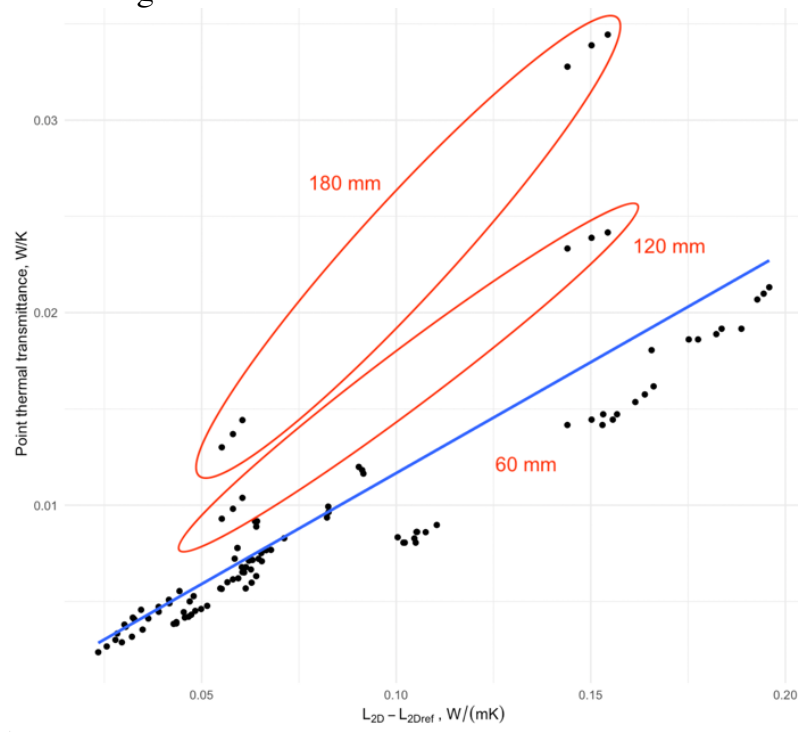

Fig. 5. Correlation between point thermal transmittance and the difference of two-dimensional thermal coupling coefficients.

It can be seen from the figure that despite reasonable correlation there are some variation depending on other input variables. The difference caused by bracket length is expected as the thermal bridge itself has more length, but other factors need further analysis. Additional thermal bridge length is used instead of point thermal transmittance to omit direct effect of bracket length and test the correlation between different input variables and the additional heat loss from the third dimension. It can be seen from figure 6 that the additional length of thermal 
bridge increases when the difference between twodimensional thermal coupling coefficients gets smaller, but the variation due to other factors is high. The variation is mainly described by the thermal conductivity of the bridging element (figure 7a) and the thermal resistance of the external (i.e. the wind barrier) layers (figure 7b).

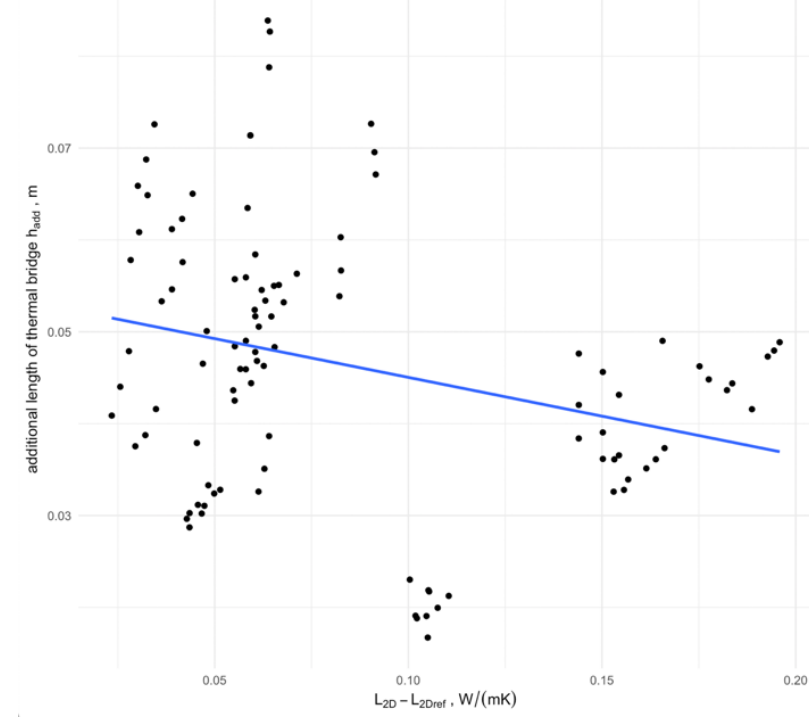

Fig. 6. Correlation between additional thermal bridge length and the difference of two-dimensional thermal coupling coefficients.
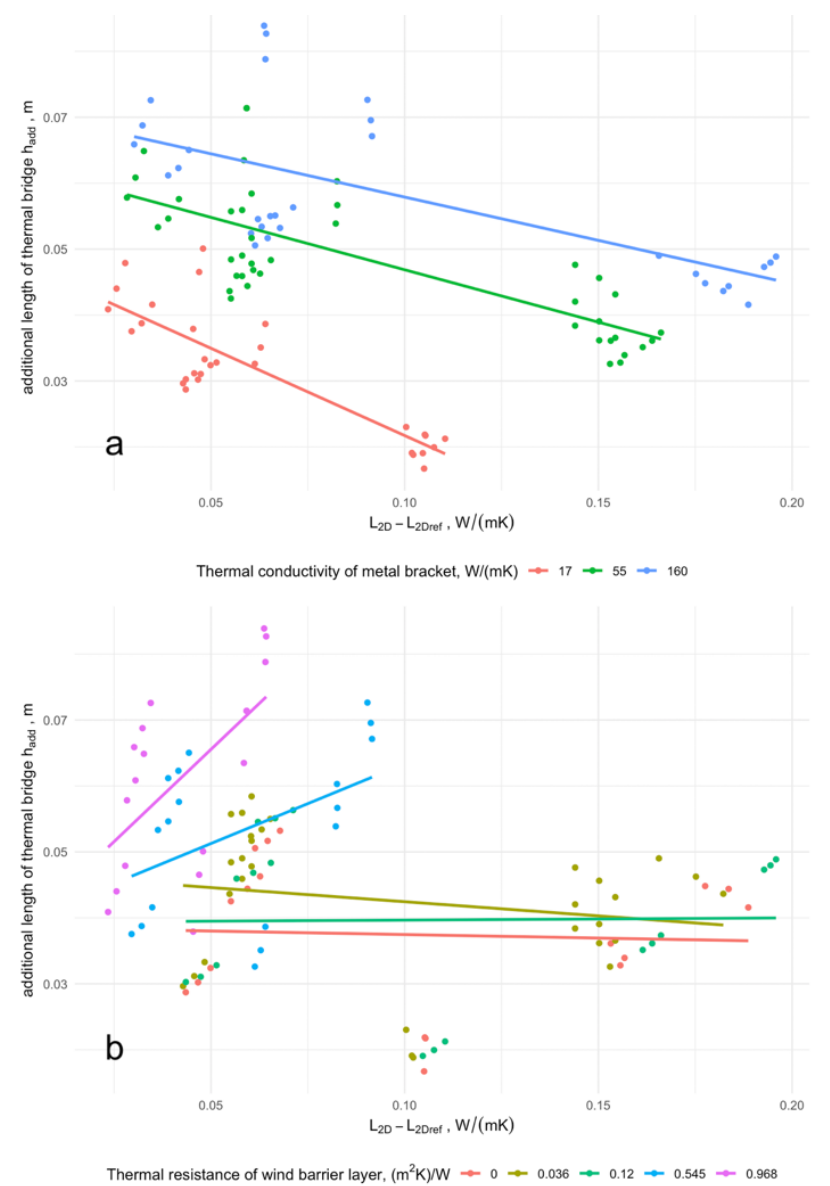

Fig. 7. Correlation between additional thermal bridge length and the difference of two-dimensional thermal coupling coefficients grouped by thermal conductivity of bridging element (a) or thermal resistance of external wind barrier layer (b).
Depending on the thermal conductivity of the bridging element the correlation between the additional length of thermal bridge and the difference of thermal coupling coefficients have roughly similar slope, but the correlation is shifted. The effect of the external wind barrier layer is different as the correlation between additional length and difference between thermal coupling coefficients is strongly affected by the thermal resistances of the outermost layers. With higher thermal resistances (mineral wool or wood fibre wind barrier) the additional length of the thermal bridge is significantly affected by the difference between respective thermal coupling coefficients. With low thermal resistance (wind barrier membrane or gypsum board) this effect is not present. This means that the variables have to be described with mutual interaction when fitting the model.

Surprisingly the thermal resistance of load bearing layer and other internal layers adjoining the thermal bridge (figure $8 \mathrm{a}$ ) and the thermal conductivity of the insulation layer with the thermal bridge (figure $8 b$ ) have no significant effect on the additional thermal bridge length.

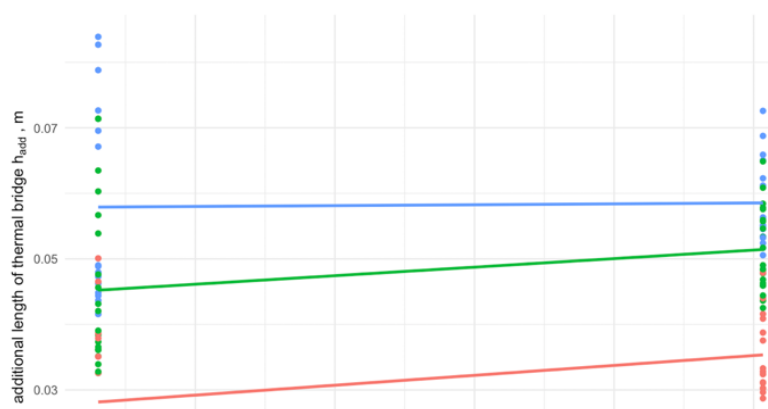

a
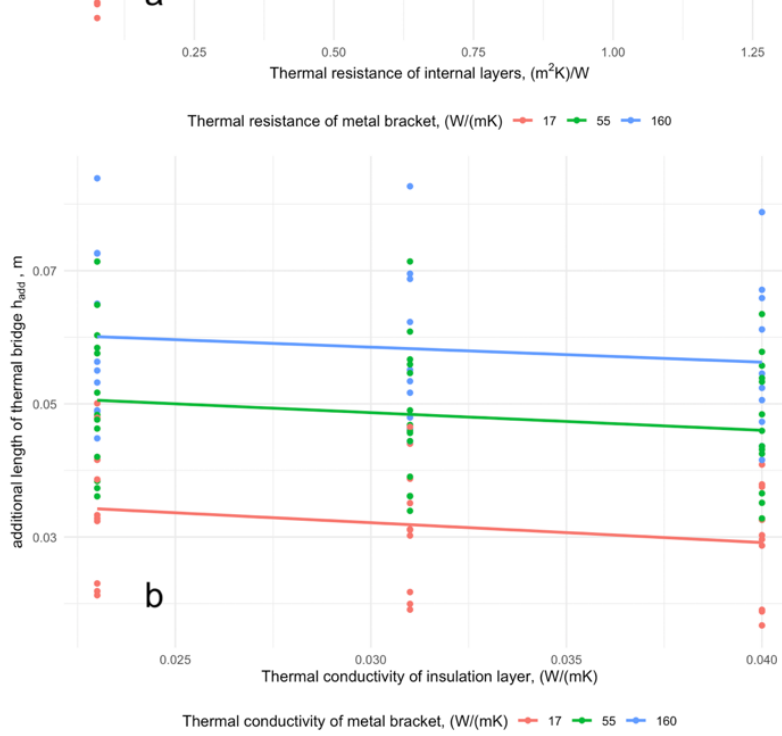

Fig. 8. Correlation between additional thermal bridge length and the thermal conductivity of insulation layer grouped by thermal conductivity of bridging element (a) or thermal resistance of external wind barrier layer (b).

Although it has been previously shown that the point thermal transmittance depends on these parameters [13] the effect is already included in the thermal coupling coefficients and the additional length of thermal bridge is 
not directly affected. This allows more simplification in the multiple regression model fitting as those parameters can be omitted.

\subsection{Model fitting}

Altogether 12 different model fits were tested to find a good balance with good fit and minimum amount of terms in multiple linear regression equation. As the difference between two-dimensional thermal coupling coefficients and the additional model length is strongly related to thermal conductivity of point thermal bridge and the thermal resistance of the wind barrier layer, it was expected that the simple correlation as in equation 5 has no predictive power (adjusted $\mathrm{R}^{2}=0.072$ ). Other model equations had better predictive power as can be seen from table 2. Although the last model equation had highest predictive power, the p-values of some coefficient estimates were a lot higher than 0.05 .

Table 2. The goodness of fit for models assessed in this study.

\begin{tabular}{|c|c|c|c|}
\hline & $\begin{array}{c}\text { Adjusted } \\
\mathrm{R}^{2}\end{array}$ & $\begin{array}{l}\text { Root mean } \\
\text { square error }\end{array}$ & $\begin{array}{c}\text { p-value for } \\
\text { coefficient } \\
\text { estimates }\end{array}$ \\
\hline $\begin{array}{c}\text { Model } \\
\text { based on } \\
\text { equation } 5\end{array}$ & 0.072 & 0.014 & $\begin{array}{c}\mathrm{c}_{1}, \mathrm{p}<0.01 \\
\mathrm{c}_{\mathrm{n}}, \mathrm{p}<0.001\end{array}$ \\
\hline $\begin{array}{c}\text { Model } \\
\text { based on } \\
\text { equation } 6\end{array}$ & 0.734 & 0.007 & $\begin{array}{c}\mathrm{c}_{1}, \mathrm{p}>0.1 \\
\mathrm{c}_{2}, \mathrm{p}<0.001 \\
\mathrm{c}_{3}, \mathrm{p}>0.1 \\
\mathrm{c}_{4}, \mathrm{p}<0.001 \\
\mathrm{c}_{\mathrm{n}}, \mathrm{p}<0.001\end{array}$ \\
\hline $\begin{array}{c}\text { Model } \\
\text { based on } \\
\text { equation } 7\end{array}$ & 0.860 & 0.005 & $\begin{array}{l}\mathrm{c}_{1}, \mathrm{p}<0.001 \\
\mathrm{c}_{2}, \mathrm{p}<0.001 \\
\mathrm{c}_{3}, \mathrm{p}<0.001 \\
\mathrm{c}_{4}, \mathrm{p}<0.001 \\
\mathrm{c}_{\mathrm{n}}, \mathrm{p}<0.001\end{array}$ \\
\hline $\begin{array}{c}\text { Model } \\
\text { based on } \\
\text { equation } 8\end{array}$ & 0.893 & 0.005 & $\begin{array}{c}\mathrm{c}_{1}, \mathrm{p}>0.1 \\
\mathrm{c}_{2}, \mathrm{p}>0.1 \\
\mathrm{c}_{3}, \mathrm{p}<0.001 \\
\mathrm{c}_{4}, \mathrm{p}<0.001 \\
\mathrm{c}_{5}, \mathrm{p}<0.001 \\
\mathrm{c}_{\mathrm{n}}, \mathrm{p}<0.001\end{array}$ \\
\hline
\end{tabular}

Final fitted equation and estimated coefficients (all parameters with $\mathrm{p}$-value $<0.001$ )

$$
\begin{aligned}
& h_{a d d}=-1.078 \cdot 10^{-1} \cdot\left(L_{2 D}-L_{2 D r e f}\right)+1.732 . \\
& 10^{-4} \cdot \lambda_{\mathrm{TB}}+1.480 \cdot 10^{-1} \cdot h_{T B}+4.162 \cdot 10^{-1} \\
& R_{e l} \cdot\left(L_{2 D}-L_{2 D r e f}\right)+2.529 \cdot 10^{-2}
\end{aligned}
$$

Estimated additional thermal bridge length can be used to calculate the point thermal transmittance of given thermal bridge as in equation 10 .

$$
\chi=\left(h_{a d d}+h_{T B}\right) \cdot\left(L_{2 D}-L_{2 D r e f}\right)
$$

Point thermal transmittances retrieved from threedimensional numerical calculations are plotted against estimated point thermal transmittances in figure 9 for the same thermal bridges.

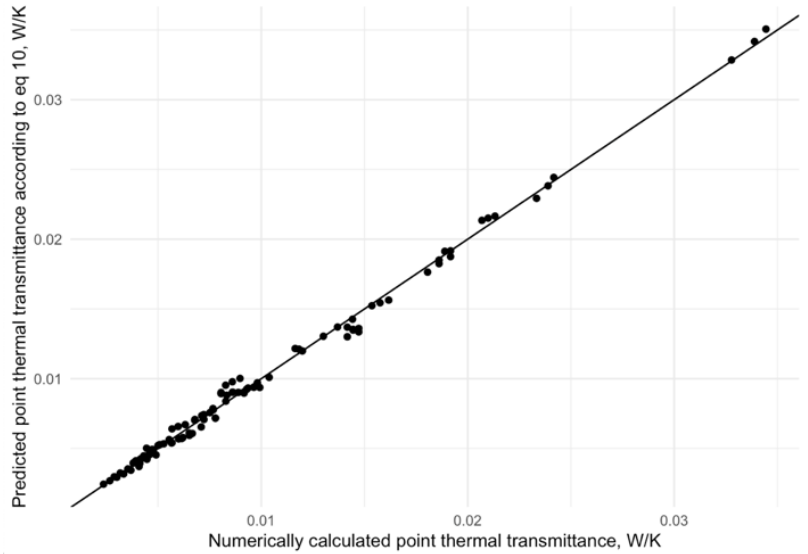

Fig. 9. Correlation between calculated and estimated point thermal transmittance (the black line represents $\mathrm{x}=\mathrm{y}$ ).

The relative error varies between $-9.2 \%$ (underestimation) and $+15.1 \%$ (overestimation) although typical deviation from the numerically calculated values were significantly lower with 0.25 and 0.75 quantiles at $2.7 \%$ and $+3.2 \%$ respectively.

The equation and the calculation methodology can be used for thermal bridges which incorporate similar situations with local point thermal bridges incorporating metal penetrations through insulation layer, such as metal brackets supporting or fixing the window frame to the load bearing structure etc. The analysis showed that the thermal conductivity of the insulation material of the layer that is penetrated and the thermal resistance of the internal (load bearing) structure has no significant effect on the equivalent length of thermal bridge, which makes it possible to use the method on wide array of different envelope configurations.

\subsection{Calculation example}

As an example, a calculation procedure according to the developed methodology is given for a plastic window connection to the lightweight steel sandwich wall panel with $160 \mathrm{~mm}$ mineral wool insulation (thermal conductivity $0.039 \mathrm{~W} /(\mathrm{mK})$ ). The point thermal bridge is created by $1 \mathrm{~mm}$ thick steel sheet that connects the internal and external layers. The length of connecting metal sheet is $200 \mathrm{~mm}$. The schematic cross section is given in figure 10 .

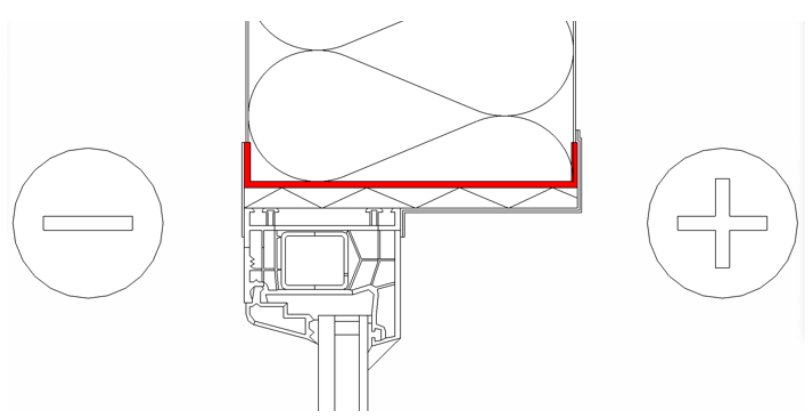

Fig. 10. Schematic cross section of assessed window connection featuring a steel penetration between window frame and sandwich wall element. 
For this junction a two-dimensional heat flow was calculated for cross section with thermal bridge element (figure 11a) and a reference case without thermal bridge element (figure 11b) by using LBNL Therm finite element calculation software [12].

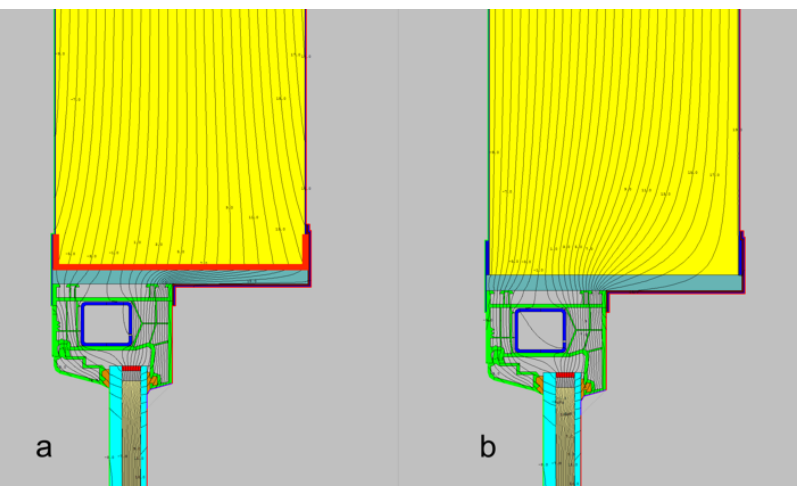

Fig. 11. Isotherms on a window connection to wall element cross section with (a) a thermal bridge and a reference case without (b) thermal bridge.

Respective thermal coupling coefficients were $L_{2 D}=$ $0.9008 \mathrm{~W} /(\mathrm{mK})$ and $L_{2 D r e f}=0.6834 \mathrm{~W} /(\mathrm{mK})$. The thermal conductivity of thermal bridge element $\lambda_{\mathrm{TB}}=50.0$ $\mathrm{W} /(\mathrm{mK})$. According to the geometric model the length of the thermal bridge is $0.200 \mathrm{~m}$ and the resistance of the wind barrier layer $R_{e l}=0\left(\mathrm{~m}^{2} \mathrm{~K}\right) / \mathrm{W}$ as thermal bridge connects directly the internal and external environment. Using equation 9 the additional thermal bridge length for this junction is $h_{\text {add }}=0.040 \mathrm{~m}$. The equivalent thermal bridge length is $h_{e q}=0.240 \mathrm{~m}$ accordingly. Multiplication with difference in thermal coupling coefficients derived from respective cross sections predicts point thermal transmittance $\chi=0.052 \mathrm{~W} / \mathrm{K}$. In comparison the point thermal transmittance derived from numerical threedimensional calculation was $\chi=0.048 \mathrm{~W} / \mathrm{K}$. For this case the point thermal transmittance was overestimated by $0.004 \mathrm{~W} / \mathrm{K}(8.3 \%)$ compared to detailed calculation following rules in ISO 10211:2017.

For comparison, in practice a very rough estimation of point thermal transmittance is sometimes calculated as the actual length of the thermal bridge $\left(h_{T B}\right)$ multiplied by the difference $\left(L_{2 D}-L_{2 D r e f}\right)$ in thermal coupling coefficients derived from two-dimensional numerical calculations based on respective cross sections. As this alternative calculation disregards the heat flow from third dimension, it always underestimates the point thermal transmittance of the bridging element. For this calculation example this rough estimation would lead to point thermal transmittance of $0.043 \mathrm{~W} / \mathrm{K}$, which underestimates the actual point thermal transmittance by $0.005 \mathrm{~W} / \mathrm{K}(10.4 \%)$. The underestimation of rough calculation is in the same magnitude as the overestimation by suggested new method for this example.

\subsection{Possible limitations of new method}

The new method is expected to work accurately for thermal bridges with small point thermal transmittance such as façade mounting brackets, fixation elements and other small metal elements inside building envelopes. The exact values of multiple linear model coefficients used in final equation are based on large number of numerical calculations for thermal bridges with point thermal transmittance in the range between 0.002 and $0.034 \mathrm{~W} / \mathrm{K}$. The equation is expected to work outside this range up to $0.2 \mathrm{~W} / \mathrm{K}$, but the estimation accuracy for significantly larger thermal bridges such as concrete columns protruding external floor elements or roof construction etc has to be systemically assessed during further research. It is expected that same underlying model structure can be used, but different model coefficients have to be derived for thermal bridges with significantly higher point thermal transmittance.

Additionally, the multiple linear regression model was based on dataset of thermal bridges with uniform geometry in third dimension. The point thermal transmittances for non-uniform bridging elements such as steel beams with C-shaped, H-shaped, L-shaped or tubular cross sections cannot be therefore predicted. The further research is planned to see if several simplifications for heat flows in third dimension can be used, such as the use of area-weighed cross sections, using additional twodimensional numerical calculations for different adjacent cross sections etc.

\section{Conclusions}

In this study a new method was proposed for predicting three-dimensional heat flow and the point thermal transmittance of thermal bridges caused by full or partial penetration of the building envelope with metal elements with uniform geometry in third dimension using twodimensional numerical calculation. This enables the possibility to estimate the point thermal transmittance of different mounting brackets etc in cases, where threedimensional calculation procedure is not possible due to lack of time, software tools or skills within design team.

A new parameter (equivalent length of thermal bridge) was defined which incorporates the effect of additional thermal transmittance when multiplied by the difference of two thermal coupling coefficients derived from twodimensional numerical calculations. The difference between equivalent length and actual length of thermal bridge can be estimated based regression model fitted to numerical data.

Altogether 102 three-dimensional cases were numerically assessed, and the effect of different parameters were analysed by varying the thermal conductivities of bridging element, insulation layer and both internal and external layers surrounding the bridging element. Based on the analysis the effect of insulation material as well as the thermal resistance of the internal layers (load bearing part of the building envelope) did not have significant effect on linear regression between the additional length of thermal bridge and the difference in thermal coupling coefficients derived from respective cross sections. Several multiple regression models were fitted to numerical data and best fit included the thermal resistance $\left(R_{e l}\right)$ of the wind barrier layers and the thermal conductivity of the bridging element itself $\left(\lambda_{\mathrm{TB}}\right)$ along 
with the actual length of the thermal bridge $\left(h_{T B}\right)$ and the difference $\left(L_{2 D}-L_{2 D r e f}\right)$ in thermal coupling coefficients derived from two-dimensional numerical calculations based on respective cross sections.

This research was supported by the Estonian Centre of Excellence in Zero Energy and Resource Efficient Smart Buildings and Districts, ZEBE (grant No. 2014-2020.4.01.150016) funded by the European Regional Development Fund, by the Estonian Research Council (grant No. PRG483), and by the European Commission through the H2020 projects Finest Twins (grant No. 856602) and NERO (grant No. 754177).

\section{References}

1. S. A. Al-Sanea and M. F. Zedan, Applied Energy 98, 584 (2012)

2. B. Berggren and M. Wall, Energy and Buildings 65, 331 (2013)

3. B. Berggren and M. Wall, Buildings 8, 154 (2018)

4. A. Capozzoli, A. Gorrino, and V. Corrado, Applied Energy 107, 229 (2013)

5. S. Ilomets, K. Kuusk, L. Paap, E. Arumägi, and T. Kalamees, Journal of Civil Engineering and Management 23, 96 (2017)

6. S. Ilomets and T. Kalamees, Journal of Building Pathology and Rehabilitation 1, 11 (2016)

7. T. Theodosiou, K. Tsikaloudaki, S. Tsoka, and P. Chastas, Journal of Cleaner Production 214, 62 (2019)

8. CEN committee ISO/TC 163/SC, ISO 10211:2017 Thermal Bridges in Building Construction. Heat Flows and Surface Temperatures. Detailed Calculations (2017)

9. ANSYS inc., (2020)

10. Comsol inc., (2020)

11. PHYSIBEL, (2020)

12. Lawrence Berkley National Laboratory, (2020)

13. T. G. Theodosiou, A. G. Tsikaloudaki, K. J. Kontoleon, and D. K. Bikas, Energy and Buildings 109, 377 (2015)

14. A. Lorenzati, S. Fantucci, A. Capozzoli, and M. Perino, Energy and Buildings 111, 164 (2016)

15. A. Ben Larbi, Energy and Buildings 37, 945 (2005)

16. R Core Team, (2018) 